\title{
Weighted dynamic inequalities of Opial-type on time scales
}

\author{
A.A. El-Deeb ${ }^{1 *}$, Fatma M. Kh², Gamal A.F. Ismail ${ }^{2}$ and Zareen A. Khan ${ }^{3}$
}

\section{"Correspondence:}

ahmedeldeeb@azhar.edu.eg

'Department of Mathematics,

Faculty of Science, Al-Azhar

University, Cairo, Egypt

Full list of author information is

available at the end of the article

\section{Springer}

\begin{abstract}
In this paper, we will state and prove some weighted dynamic inequalities of Opial-type involving integrals of powers of a function and of its derivative on time scales which not only extend some results in the literature but also improve some of them. The main results will be proved by using some algebraic inequalities, the Hölder inequality and a simple consequence of Keller's chain rule on time scales. As special cases of the obtained dynamic inequalities, we will get some continuous and discrete inequalities.
\end{abstract}

MSC: 26D10;26D15;26E70; 34A40

Keywords: Opial-type inequality; Dynamic inequality; Time scale

\section{Introduction}

In 1960, the Polish Mathematician Opial [36] proved an inequality involving integrals of functions and their derivatives;

$$
\int_{a}^{b}|x(t)|\left|x^{\prime}(t)\right| d t \leq \frac{b-a}{4} \int_{a}^{b}\left|x^{\prime}(t)\right|^{2} d t,
$$

where $x$ is an absolutely differentiable continuous function on $[a, b], x(a)=x(b)=0, x(t)>$ 0 , and the constant $\frac{b-a}{4}$ is sharp, in the sense that $\frac{b-a}{4}$ cannot be replaced by a smaller constant.

Since the publication of the above result in 1960, numerous papers with new evidence, different speculations, and augmentations have showed up in the literature. Inequalities which involve integrals of functions and their derivatives are of great importance in mathematics with applications in the theory of differential equations, approximations and probability $[1-4,7,17,18,21,22,28,29,34]$.

As a generalization of (1.1), Beesack [10] proved that: If $x$ is an absolutely continuous function on $[a, b]$ with $x(a)=0$, then

$$
\int_{a}^{b}|x(t)|\left|x^{\prime}(t)\right| d t \leq \frac{1}{2} \int_{a}^{b} \frac{1}{r(t)} d t \int_{a}^{b} r(t)\left|x^{\prime}(t)\right|^{2} d t
$$

where $r$ is a positive and continuous function with $\int_{a}^{b} \frac{d t}{r(t)}<\infty$.

(C) The Author(s) 2019. This article is distributed under the terms of the Creative Commons Attribution 4.0 International License (http://creativecommons.org/licenses/by/4.0/), which permits unrestricted use, distribution, and reproduction in any medium, provided you give appropriate credit to the original author(s) and the source, provide a link to the Creative Commons license, and indicate if changes were made. 
Yang [44] simplified the Beesack proof and extended the inequality (1.2) as follows: If $x$ is an absolutely continuous function on $(a, b)$ with $x(a)=0$, then

$$
\int_{a}^{b} q(t)|x(t)|\left|x^{\prime}(t)\right| d t \leq \frac{1}{2} \int_{a}^{b} \frac{1}{r(t)} d t \int_{a}^{b} r(t) q(t)\left|x^{\prime}(t)\right|^{2} d t
$$

where $r$ is a positive and continuous function with $\int_{a}^{b} \frac{d t}{r(t)}<\infty$ and $q$ is a positive, bounded, and nonincreasing function on $[a, b]$.

Recently, the theory of time scales, which has been initiated by Stefen Hilger in his Ph.D. thesis [30] in order to unify discrete and continuous analysis, has gained a lot of attention. During the previous decade, an impressive number of dynamic imbalances have been given by numerous creators who were inspired by certain applications (see $[5,6,9,12,13$, $16,19,20,23-27,31,35,37,39,41])$. The general thought is to demonstrate a result for a dynamic inequality where the domain of the unknown function is a so-called time scale $\mathbb{T}$, which is an arbitrary nonempty closed subset of real numbers. The three best-known time scales are $\mathbb{T}=\mathbb{R}, \mathbb{T}=\mathbb{Z}$ and $\mathbb{T}=\overline{q^{\mathbb{Z}}}=\left\{q^{z}: z \in \mathbb{Z}\right\} \cup\{0\}$ where $q>1$. The books [14] and [15] organize and summarize much of time scales calculus.

In [11], Bohner and Kaymakçalan introduced a dynamic Opial inequality which extended the continuous version inequality (1.1) to a general time scale and studied if $x:[a, b] \cap \mathbb{T} \longrightarrow \mathbb{R}$ is delta differentiable with $x(a)=0$, then

$$
\int_{a}^{b}\left|x(t)+x^{\sigma}(t)\right|\left|x^{\Delta}(t)\right| \Delta t \leq(b-a) \int_{a}^{b}\left|x^{\Delta}(t)\right|^{2} \Delta t .
$$

Dynamic Opial's inequalities on time scales got a lot of consideration and numerous papers have been composed; see [11,33, 38, 40, 42, 43] and the references cited therein.

Also in [11] the authors extended the inequality (1.3) of Yang and proved that: If $r$ and $q$ are positive rd-continuous functions on $[a, b]_{\mathbb{T}}, \int_{a}^{b} \frac{\Delta t}{r(t)}<\infty, q$ is nonincreasing and $x$ : $[a, b] \cap \mathbb{T} \longrightarrow \mathbb{R}$ is delta differentiable with $x(a)=0$, then

$$
\int_{a}^{b} q^{\sigma}(t)\left|x(t)+x^{\sigma}(t)\right|\left|x^{\Delta}(t)\right| \Delta t \leq \int_{a}^{b} \frac{\Delta t}{r(t)} \int_{a}^{b} r(t) q(t)\left|x^{\Delta}(t)\right|^{2} \Delta t
$$

Karpuz et al. [33] established the same inequality as in (1.5) by replacing $q^{\sigma}$ with $q$ of the form

$$
\int_{a}^{b} q(t)\left|x(t)+x^{\sigma}(t)\right|\left|x^{\Delta}(t)\right| \Delta t \leq K_{q}(a, b) \int_{a}^{b}\left|x^{\Delta}(t)\right|^{2} \Delta t
$$

where $q$ is a positive rd-continuous function on $[a, b]_{\mathbb{T}}, x:[a, b] \cap \mathbb{T} \rightarrow \mathbb{R}$ is delta differentiable with $x(a)=a$, and

$$
K_{q}(a, b)=\left(2 \int_{a}^{b} q^{2}(u)(\sigma(u)-a) \Delta u\right)^{\frac{1}{2}} .
$$

For $p \geq 1$, Karpuz and Özkan [32] proved that: If $y:[a, \tau] \cap \mathbb{T} \rightarrow \mathbb{R}^{+}$is delta differentiable with $y(a)=0$ and $y^{\Delta}$ does not change sign in $(a, \tau)_{\mathbb{T}}$, then we have

$$
\int_{a}^{\tau} s(x)\left|y(x)+y^{\sigma}(x)\right|^{p}\left|y^{\Delta}(x)\right|^{q} \Delta x \leq K_{1}(a, \tau, p, q) \int_{a}^{\tau} r(x)\left|y^{\Delta}(x)\right|^{p+q} \Delta x,
$$


where

$$
\begin{aligned}
K_{1}(a, \tau, p, q)= & 2^{2 p-1}\left(\frac{q}{p+q}\right)^{\frac{q}{p+q}}\left[\int_{a}^{\tau} s^{\frac{p+q}{p}}(x)\left(\frac{1}{r(x)}\right)^{\frac{q}{p}}\left(\int_{a}^{x} \frac{1}{r^{\frac{1}{p+q-1}}(t)} \Delta t\right)^{p+q-1} \Delta x\right]^{\frac{p}{p+q}} \\
& +2^{p-1} \max _{a \leq x \leq \tau}\left(\frac{\mu^{p}(x) s(x)}{r(x)}\right)
\end{aligned}
$$

$p, q$ are positive real numbers such that $p \geq 1$, and $r, s$ are nonnegative rd-continuous functions on $(a, \tau)_{\mathbb{T}}$ such that $\int_{a}^{\tau} r^{\frac{-1}{p+q-1}}(t) \Delta t<\infty$.

In the same paper, the authors proved that: If $y:[\tau, b] \cap \mathbb{T} \rightarrow \mathbb{R}^{+}$is delta differentiable with $y(b)=0$ and $y^{\Delta}$ does not change sign in $(\tau, b)_{\mathbb{T}}$, then we have

$$
\int_{\tau}^{b} s(x)\left|y(x)+y^{\sigma}(x)\right|^{p}\left|y^{\Delta}(x)\right|^{q} \Delta x \leq K_{2}(\tau, b, p, q) \int_{\tau}^{b} r(x)\left|y^{\Delta}(x)\right|^{p+q} \Delta x
$$

where

$$
\begin{aligned}
K_{2}(\tau, b, p, q)= & 2^{2 p-1}\left(\frac{q}{p+q}\right)^{\frac{q}{p+q}}\left[\int_{\tau}^{b} s^{\frac{p+q}{p}}(x)\left(\frac{1}{r(x)}\right)^{\frac{q}{p}}\left(\int_{x}^{b} \frac{1}{r^{\frac{1}{p+q-1}}(t)} \Delta t\right)^{p+q-1} \Delta x\right]^{\frac{p}{p+q}} \\
& +2^{p-1} \max _{\tau \leq x \leq b}\left(\frac{\mu^{p}(x) s(x)}{r(x)}\right),
\end{aligned}
$$

$p, q$ are positive real numbers such that $p \geq 1$, and $r, s$ are nonnegative rd-continuous functions on $(\tau, b)_{\mathbb{T}}$ such that $\int_{\tau}^{b} r^{\frac{-1}{p+q-1}}(t) \Delta t<\infty$.

Adding (1.7) and (1.8), Karpus and Özkan proved that: If $y:[a, b] \cap \mathbb{T} \rightarrow \mathbb{R}^{+}$is delta differentiable with $y(a)=y(b)=0$, then

$$
\int_{a}^{b} s(x)\left|y(x)+y^{\sigma}(x)\right|^{p}\left|y^{\Delta}(x)\right|^{q} \Delta x \leq K(p, q) \int_{a}^{b} r(x)\left|y^{\Delta}(x)\right|^{p+q} \Delta x,
$$

where

$$
K(p, q)=K_{1}(a, \tau, p, q)=K_{2}(\tau, b, p, q) .
$$

For $p \leq 1$, Karpuz and Özkan [32] proved that: If $y:[a, \tau] \cap \mathbb{T} \rightarrow \mathbb{R}^{+}$is delta differentiable with $y(a)=0$ and $y^{\Delta}$ does not change sign in $(a, \tau)_{\mathbb{T}}$, then we have

$$
\int_{a}^{\tau} s(x)\left|y(x)+y^{\sigma}(x)\right|^{p}\left|y^{\Delta}(x)\right|^{q} \Delta x \leq K_{3}(a, \tau, p, q) \int_{a}^{\tau} r(x)\left|y^{\Delta}(x)\right|^{p+q} \Delta x
$$

where

$$
K_{3}(a, \tau, p, q)=\frac{K_{1}(a, \tau, p, q)}{2^{2 p-1}},
$$

$p, q$ are positive real numbers such that $p \leq 1, p+q>1$ and $r, s$ are nonnegative rdcontinuous functions on $(a, \tau)_{\mathbb{T}}$ such that $\int_{a}^{\tau} r^{\frac{-1}{p+q-1}}(t) \Delta t<\infty$. 
Also, in the same paper, the authors proved that: If $y:[\tau, b] \cap \mathbb{T} \rightarrow \mathbb{R}^{+}$is delta differentiable with $y(b)=0$ and $y^{\Delta}$ does not change sign in $(\tau, b)_{\mathbb{T}}$, then we have

$$
\int_{\tau}^{b} s(x)\left|y(x)+y^{\sigma}(x)\right|^{p}\left|y^{\Delta}(x)\right|^{q} \Delta x \leq K_{4}(\tau, b, p, q) \int_{\tau}^{b} r(x)\left|y^{\Delta}(x)\right|^{p+q} \Delta x,
$$

where

$$
K_{4}(\tau, b, p, q)=\frac{K_{2}(\tau, b, p, q)}{2^{2 p-1}},
$$

$p, q$ are positive real numbers such that $p \leq 1, p+q>1$ and $r, s$ are nonnegative rdcontinuous functions on $(\tau, b)_{\mathbb{T}}$ such that $\int_{\tau}^{b} r^{\frac{-1}{p+q-1}}(t) \Delta t<\infty$.

Combining (1.10) and (1.11), Karpus and Özkan proved that: If $y:[a, b] \cap \mathbb{T} \rightarrow \mathbb{R}^{+}$is delta differentiable with $y(a)=y(b)=0$, then

$$
\int_{a}^{b} s(x)\left|y(x)+y^{\sigma}(x)\right|^{p}\left|y^{\Delta}(x)\right|^{q} \Delta x \leq K_{1}^{\star}(p, q) \int_{a}^{b} r(x)\left|y^{\Delta}(x)\right|^{p+q} \Delta x,
$$

where

$$
K_{1}^{\star}(p, q)=K_{3}(a, \tau, p, q)=K_{4}(\tau, b, p, q) .
$$

In this article, motivated by the above inequalities, we will explore some dynamic Opialtype inequalities on time scales, which generalize inequalities (1.7)-(1.12). After each result, we will study the special cases when $\mathbb{T}=\mathbb{R}$ and $\mathbb{T}=\mathbb{N}$ to obtain some continuous and discrete results.

\section{Basics of time scales}

Firstly, we recall some essentials of time scales, and some universal symbols that will be used in the present paper. From now on, $\mathbb{R}$ and $\mathbb{Z}$ are the set of real numbers and the set of integers, respectively.

A time scale $\mathbb{T}$ is an arbitrary nonempty closed subset of the set of real numbers $\mathbb{R}$. Throughout the article, we assume that $\mathbb{T}$ has the topology that it inherits from the standard topology on $\mathbb{R}$. We define the forward jump operator $\sigma: \mathbb{T} \rightarrow \mathbb{T}$ for any $t \in \mathbb{T}$ by

$$
\sigma(t):=\inf \{s \in \mathbb{T}: s>t\}
$$

and the backward jump operator $\rho: \mathbb{T} \rightarrow \mathbb{T}$ for any $t \in \mathbb{T}$ by

$$
\rho(t):=\sup \{s \in \mathbb{T}: s<t\}
$$

In the preceding two definitions, we set $\inf \emptyset=\sup \mathbb{T}$ (i.e., if $t$ is the maximum of $\mathbb{T}$, then $\sigma(t)=t$ ) and $\sup \emptyset=\inf \mathbb{T}$ (i.e., if $t$ is the minimum of $\mathbb{T}$, then $\rho(t)=t$ ), where $\emptyset$ denotes the empty set.

A point $t \in \mathbb{T}$ with $\inf \mathbb{T}<t<\sup \mathbb{T}$ is said to be right-scattered if $\sigma(t)>t$, right-dense if $\sigma(t)=t$, left-scattered if $\rho(t)<t$, and left-dense if $\rho(t)=t$. Points that are simultaneously right-dense and left-dense are said to be dense points. Points that are simultaneously rightscattered and left-scattered are said to be isolated points. 
The forward graininess function $\mu: \mathbb{T} \rightarrow[0, \infty)$ is defined for any $t \in \mathbb{T}$ by $\mu(t):=\sigma(t)-t$ and the backward graininess function $v: \mathbb{T} \rightarrow[0, \infty)$ is defined for any $t \in \mathbb{T}$ by $v(t):=$ $t-\rho(t)$.

If $f: \mathbb{T} \rightarrow \mathbb{R}$ is a function, then the function $f^{\sigma}: \mathbb{T} \rightarrow \mathbb{R}$ is defined by $f^{\sigma}(t)=f(\sigma(t)), \forall t \in$ $\mathbb{T}$, that is, $f^{\sigma}=f \circ \sigma$. Similarly, the function $f^{\rho}: \mathbb{T} \rightarrow \mathbb{R}$ is defined by $f^{\rho}(t)=g(\rho(t)), \forall t \in \mathbb{T}$, that is, $f^{\rho}=f \circ \rho$.

The sets $\mathbb{T}^{\kappa}, \mathbb{T}_{\kappa}$ and $\mathbb{T}_{\kappa}^{\kappa}$ are introduced as follows: If $\mathbb{T}$ has a left-scattered maximum $t_{1}$, then $\mathbb{T}^{\kappa}=\mathbb{T}-\left\{t_{1}\right\}$, otherwise $\mathbb{T}^{\kappa}=\mathbb{T}$. If $\mathbb{T}$ has a right-scattered minimum $t_{2}$, then $\mathbb{T}^{\kappa}=$ $\mathbb{T}-\left\{t_{2}\right\}$, otherwise $\mathbb{T}_{\kappa}=\mathbb{T}$. Finally, we have $\mathbb{T}_{\kappa}^{\kappa}=\mathbb{T}^{\kappa} \cap \mathbb{T}_{\kappa}$.

The interval $[a, b]$ in $\mathbb{T}$ is defined by

$$
[a, b]_{\mathbb{T}}=\{t \in \mathbb{T}: a \leq t \leq b\}
$$

We define the open intervals and half-closed intervals similarly.

Assume $f: \mathbb{T} \rightarrow \mathbb{R}$ is a function and $t \in \mathbb{T}^{\kappa}$. Then $f^{\Delta}(t) \in \mathbb{R}$ is said to be the delta derivative of $f$ at $t$ if for any $\varepsilon>0$ there exists a neighborhood $U$ of $t$ such that, for every $s \in U$, we have

$$
\mid f(\sigma(t))-f(s)]-f^{\Delta}(t)[\sigma(t)-s]|\leq \varepsilon| \sigma(t)-s \mid .
$$

Moreover, $f$ is said to be delta differentiable on $\mathbb{T}^{\kappa}$ if it is delta differentiable at every $t \in \mathbb{T}^{\kappa}$.

Similarly, we say that $f^{\nabla}(t) \in \mathbb{R}$ is the nabla derivative of $f$ at $t$ if for any $\varepsilon>0$ there exists a neighborhood $V$ of $t$ such that for all $s \in V$

$$
\left|[f(\rho(t))-f(s)]-f^{\nabla}(t)[\rho(t)-s]\right| \leq \varepsilon|\rho(t)-s| .
$$

Furthermore, $f$ is said to be nabla differentiable on $\mathbb{T}_{\kappa}$ if it is nabla differentiable at each $t \in \mathbb{T}_{\kappa}$.

A function $f: \mathbb{T} \rightarrow \mathbb{R}$ is said to be right-dense continuous (rd-continuous) if $f$ is continuous at all right-dense points in $\mathbb{T}$ and its left-sided limits exist at all left-dense points in $\mathbb{T}$.

In a similar manner, a function $f: \mathbb{T} \rightarrow \mathbb{R}$ is said to be left-dense continuous (ldcontinuous) if $f$ is continuous at all left-dense points in $\mathbb{T}$ and its right-sided limits exist at all right-dense points in $\mathbb{T}$.

The delta integration by parts on time scales is given by the following formula:

$$
\int_{a}^{b} g^{\Delta}(t) f(t) \Delta t=g(b) f(b)-g(a) f(a)-\int_{a}^{b} g^{\sigma}(t) f^{\Delta}(t) \Delta t,
$$

whereas the nabla integration by parts on time scales is given by

$$
\int_{a}^{b} g^{\nabla}(t) f(t) \nabla t=g(b) f(b)-g(a) f(a)-\int_{a}^{b} g^{\rho}(t) f^{\nabla}(t) \nabla t .
$$

We will use the following crucial relations between calculus on time scales $\mathbb{T}$ and either differential calculus on $\mathbb{R}$ or difference calculus on $\mathbb{Z}$. Note that: 
(i) If $\mathbb{T}=\mathbb{R}$, then

$$
\begin{aligned}
& \sigma(t)=\rho(t)=t \\
& \mu(t)=v(t)=0 \\
& f^{\Delta}(t)=f^{\nabla}(t)=f^{\prime}(t), \\
& \int_{a}^{b} f(t) \Delta t=\int_{a}^{b} f(t) \nabla t=\int_{a}^{b} f(t) d t .
\end{aligned}
$$

(ii) If $\mathbb{T}=\mathbb{Z}$, then

$$
\begin{aligned}
& \sigma(t)=t+1, \\
& \rho(t)=t-1, \\
& \mu(t)=v(t)=1, \\
& f^{\Delta}(t)=\Delta f(t), \\
& f^{\nabla}(t)=\nabla f(t), \\
& \int_{a}^{b} f(t) \Delta t=\sum_{t=a}^{b-1} f(t), \\
& \int_{a}^{b} f(t) \nabla t=\sum_{t=a+1}^{b} f(t),
\end{aligned}
$$

where $\Delta$ and $\nabla$ are the forward and backward difference operators, respectively.

\section{Main results}

In this section, we will state and prove our main results.

First, we present the basic theorems that will be needed in the proof of our main results.

Theorem 3.1 (Chain rule on time scales [14]) Assume $g: \mathbb{R} \rightarrow \mathbb{R}$ is continuous, $g: \mathbb{T} \rightarrow \mathbb{R}$ is delta differentiable on $\mathbb{T}^{\kappa}$, and $f: \mathbb{R} \rightarrow \mathbb{R}$ is continuously differentiable. Then there exists $c \in[t, \sigma(t)]$ with

$$
(f \circ g)^{\Delta}(t)=f^{\prime}(g(c)) g^{\Delta}(t)
$$

Theorem 3.2 (Chain rule on time scales [14]) Let $f: \mathbb{R} \rightarrow \mathbb{R}$ be continuously differentiable and suppose $g: \mathbb{T} \rightarrow \mathbb{R}$ is delta differentiable. Then $f \circ g: \mathbb{T} \rightarrow \mathbb{R}$ is delta differentiable and the formula

$$
(f \circ g)^{\Delta}(t)=\left\{\int_{0}^{1}\left[f^{\prime}\left(h g^{\sigma}(t)+(1-h) g(t)\right)\right] d h\right\} g^{\Delta}(t)
$$

holds. 
Theorem 3.3 (Dynamic Hölder inequality [14]) Let $a, b \in \mathbb{T}$ and $f, g \in C_{r d}\left([a, b]_{\mathbb{T}},[0, \infty)\right)$. If $p, q>1$ with $\frac{1}{p}+\frac{1}{q}=1$, then

$$
\int_{a}^{b} f(t) g(t) \Delta t \leq\left[\int_{a}^{b} f^{p}(t) \Delta t\right]^{\frac{1}{p}}\left[\int_{a}^{b} g^{q}(t) \Delta t\right]^{\frac{1}{q}}
$$

Also, the main results here will be proved by employing the inequalities (see [8], page 51)

$$
\begin{aligned}
& a^{\lambda}+b^{\lambda} \leq(a+b)^{\lambda} \leq 2^{\lambda-1}\left(a^{\lambda}+b^{\lambda}\right), \quad \text { if } a, b \geq 0, \lambda \geq 1 ; \\
& a^{\lambda}+b^{\lambda} \geq(a+b)^{\lambda} \geq 2^{\lambda-1}\left(a^{\lambda}+b^{\lambda}\right), \quad \text { if } a, b \geq 0,0 \leq \lambda \leq 1 .
\end{aligned}
$$

Next, we enlist the following assumptions for the proofs of our main results:

(A1) $\mathbb{T}$ be a time scale with (i) $a, \tau \in \mathbb{T}$; (ii) $\tau, b \in \mathbb{T}$; (iii) $a, b \in \mathbb{T}$.

(A2) $p$, $q$ be positive real numbers such that (i) $p \geq 1$; (ii) $p \leq 1$; (iii) $p+q>1$.

(A3) $r, s$ be nonnegative rd-continuous functions on (i) $(a, \tau)_{\mathbb{T}}$ provided that $\int_{a}^{\tau} r^{\frac{-1}{p+q-1}}(t) \Delta t<\infty$; (ii) $(\tau, b)_{\mathbb{T}}$ such that $\int_{\tau}^{b} r^{\frac{-1}{p+q-1}}(t) \Delta t<\infty$; (iii) $(a, b)_{\mathbb{T}}$ with $\int_{a}^{b} r^{\frac{-1}{p+q-1}}(t) \Delta t<\infty$.

(A4) $y:[a, \tau] \cap \mathbb{T} \rightarrow \mathbb{R}^{+}$is delta differentiable with $y(a) \neq 0$ and $y^{\Delta}$ does not change sign in $(a, \tau)_{\mathbb{T}}$.

(A5) $y:[\tau, b] \cap \mathbb{T} \rightarrow \mathbb{R}^{+}$be delta differentiable such that $y(b) \neq 0$ and $y^{\Delta}$ does not change sign in $(\tau, b)_{\mathbb{T}}$.

(A6) $y:[a, b] \cap \mathbb{T} \rightarrow \mathbb{R}^{+}$is delta differentiable and $y(a) \neq 0, y(b) \neq 0$. Also $y^{\Delta}$ does not change sign in $(a, b)_{\mathbb{T}}$.

(A7) $\left\{r_{i}\right\}_{0 \leq i \leq N}$ and $\left\{s_{i}\right\}_{0 \leq i \leq N}$ are nonnegative real sequences.

(A8) $\left\{y_{i}\right\}_{0 \leq i \leq N}$ is a sequence of real numbers with (i) $y(a) \neq 0$; (ii) $y(b) \neq 0$; (iii) $y(a) \neq 0$ and $y(b) \neq 0$.

Now, we are ready to state and prove the first result, which generalizes many inequalities in the literature.

Theorem 3.4 Let $(A 1)(i),(A 2)(i),(A 3)(i)$ and $(A 4)$ be satisfied.

(a) Then

$$
\begin{aligned}
& \int_{a}^{\tau} s(x)\left|y(x)+y^{\sigma}(x)\right|^{p}\left|y^{\Delta}(x)\right|^{q} \Delta x \\
& \quad \leq K_{5}(a, \tau, p, q) \int_{a}^{\tau} r(x)\left|y^{\Delta}(x)\right|^{p+q} \Delta x+2^{3 p-2}|y(a)|^{p} \int_{a}^{\tau} s(x)\left|y^{\Delta}(x)\right|^{q} \Delta x,
\end{aligned}
$$

where

$$
\begin{aligned}
& K_{5}(a, \tau, p, q) \\
& =2^{3 p-2}\left(\frac{q}{p+q}\right)^{\frac{q}{p+q}}\left[\int_{a}^{\tau} s^{\frac{p+q}{p}}(x)\left(\frac{1}{r(x)}\right)^{\frac{q}{p}}\left(\int_{a}^{x} \frac{1}{r^{\frac{1}{p+q-1}}(t)} \Delta t\right)^{p+q-1} \Delta x\right]^{\frac{p}{p+q}} \\
& \quad+2^{p-1} \max _{a \leq x \leq \tau}\left(\frac{\mu^{p}(x) s(x)}{r(x)}\right) .
\end{aligned}
$$


(b) If $r=s$, then

$$
\begin{aligned}
& \int_{a}^{\tau} r(x)\left|y(x)+y^{\sigma}(x)\right|^{p}\left|y^{\Delta}(x)\right|^{q} \Delta x \\
& \quad \leq K_{6}(a, \tau, p, q) \int_{a}^{\tau} r(x)\left|y^{\Delta}(x)\right|^{p+q} \Delta x+2^{3 p-2}|y(a)|^{p} \int_{a}^{\tau} r(x)\left|y^{\Delta}(x)\right|^{q} \Delta x
\end{aligned}
$$

where

$$
\begin{aligned}
K_{6}(a, \tau, p, q)= & 2^{3 p-2}\left(\frac{q}{p+q}\right)^{\frac{q}{p+q}}\left[\int_{a}^{\tau} r(x)\left(\int_{a}^{x} \frac{1}{r^{\frac{1}{p+q-1}}(t)} \Delta t\right)^{p+q-1} \Delta x\right]^{\frac{p}{p+q}} \\
& +2^{p-1} \max _{a \leq x \leq \tau}\left(\mu^{p}(x)\right) .
\end{aligned}
$$

(c) Let $r=1$. Then

$$
\begin{aligned}
& \int_{a}^{\tau}\left|y(x)+y^{\sigma}(x)\right|^{p}\left|y^{\Delta}(x)\right|^{q} \Delta x \\
& \quad \leq K_{7}(a, \tau, p, q) \int_{a}^{\tau}\left|y^{\Delta}(x)\right|^{p+q} \Delta x+2^{3 p-2}|y(a)|^{p} \int_{a}^{\tau}\left|y^{\Delta}(x)\right|^{q} \Delta x,
\end{aligned}
$$

where

$$
K_{7}(a, \tau, p, q)=\left(2^{3 p-2} \frac{q^{\frac{q}{p+q}}}{p+q}(\tau-a)^{p}+2^{p-1} \max _{a \leq x \leq \tau}\left(\mu^{p}(x)\right)\right) .
$$

Proof (a) Since $y^{\Delta}$ does not change sign in $(a, \tau)_{\mathbb{T}}$, we have

$$
|y(x)|-|y(a)| \leq|y(x)-y(a)|=\left|\int_{a}^{x} y^{\Delta}(t) \Delta t\right| \leq \int_{a}^{x}\left|y^{\Delta}(t)\right| \Delta t .
$$

From (3.10), we get

$$
|y(x)| \leq \int_{a}^{x}\left|y^{\Delta}(t)\right| \Delta t+|y(a)|=\int_{a}^{x} \frac{1}{r^{\frac{1}{p+q}}(t)} r^{\frac{1}{p+q}}(t)\left|y^{\Delta}(t)\right| \Delta t+|y(a)| .
$$

Now, since $r$ is nonnegative on $(a, \tau)_{\mathbb{T}}$, it follows from the Hölder inequality (3.3) with indices $\frac{p+q}{p+q-1}$ and $p+q$, and with

$$
f(t)=\frac{1}{r^{\frac{1}{p+q}}(t)}, \quad g(t)=r^{\frac{1}{p+q}}(t)\left|y^{\Delta}(t)\right|,
$$

that

$$
|y(x)| \leq\left(\int_{a}^{x} \frac{1}{r^{\frac{1}{p+q-1}}(t)} \Delta t\right)^{\frac{p+q-1}{p+q}}\left(\int_{a}^{x} r(t)\left|y^{\Delta}(t)\right|^{p+q} \Delta t\right)^{\frac{1}{p+q}}+|y(a)| .
$$

Since $p \geq 1$, by taking the power $p$ for both sides of (3.11), we have

$$
|y(x)|^{p} \leq\left[\left(\int_{a}^{x} \frac{1}{r^{\frac{1}{p+q-1}}(t)} \Delta t\right)^{\frac{p+q-1}{p+q}}\left(\int_{a}^{x} r(t)\left|y^{\Delta}(t)\right|^{p+q} \Delta t\right)^{\frac{1}{p+q}}+|y(a)|\right]^{p} .
$$


Applying the inequality (3.4) on the right-hand side of (3.12), we deduce

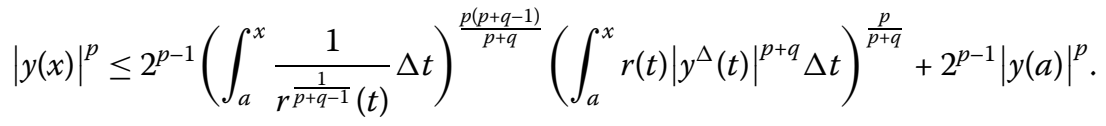

Since $y^{\sigma}=y+\mu y^{\Delta}$, we have

$$
y(x)+y^{\sigma}(x)=2 y(x)+\mu y^{\Delta}(x) .
$$

Obviously, $p \geq 1$. Taking power $p$ for both sides of (3.13) and using the inequality (3.4), we deduce

$$
\begin{aligned}
\left|y(x)+y^{\sigma}(x)\right|^{p} & \leq 2^{p-1}\left(2^{p}|y(x)|^{p}+\mu^{p}(x)\left|y^{\Delta}(x)\right|^{p}\right) \\
& =2^{2 p-1}|y(x)|^{p}+2^{p-1} \mu^{p}(x)\left|y^{\Delta}(x)\right|^{p} .
\end{aligned}
$$

Setting

$$
z(x):=\int_{a}^{x} r(t)\left|y^{\Delta}(t)\right|^{p+q} \Delta t
$$

we see that $z(a)=0$, and

$$
z^{\Delta}(x)=r(x)\left|y^{\Delta}(x)\right|^{p+q}>0
$$

From (3.16), we get

$$
\left|y^{\Delta}(x)\right|^{p+q}=\frac{z^{\Delta}(x)}{r(x)} \text { and }\left|y^{\Delta}(x)\right|^{q}=\left(\frac{z^{\Delta}(x)}{r(x)}\right)^{\frac{q}{p+q}}
$$

From (3.14), (3.17) and since $s$ is nonnegative on $(a, \tau)_{\mathbb{T}}$, we have

$$
\begin{aligned}
& s(x)\left|y(x)+y^{\sigma}(x)\right|^{p}\left|y^{\Delta}(x)\right|^{q} \\
& \leq 2^{2 p-1} s(x)|y(x)|^{p}\left|y^{\Delta}(x)\right|^{q}+2^{p-1} \mu^{p}(x) s(x)\left|y^{\Delta}\right|^{p+q} \\
& \leq 2^{3 p-2} s(x)\left(\frac{1}{r(x)}\right)^{\frac{q}{p+q}}\left(\int_{a}^{x} \frac{1}{r^{\frac{1}{p+q-1}}(t)} \Delta t\right)^{\frac{p(p+q-1)}{p+q}} \\
& \quad \times z^{\frac{p}{p+q}}(x)\left(z^{\Delta}(x)\right)^{\frac{q}{p+q}}+2^{3 p-2} s(x)|y(a)|^{p}\left(\frac{z^{\Delta}(x)}{r(x)}\right)^{\frac{q}{p+q}} \\
& \quad+2^{p-1} \mu^{p}(x) s(x) \frac{z^{\Delta}(x)}{r(x)} .
\end{aligned}
$$

Integrating the above inequality from $a$ to $\tau$, we get

$$
\begin{aligned}
& \int_{a}^{\tau} s(x)\left|y(x)+y^{\sigma}(x)\right|^{p}\left|y^{\Delta}(x)\right|^{q} \Delta x \\
& \quad \leq 2^{3 p-2} \int_{a}^{\tau} s(x)\left(\frac{1}{r(x)}\right)^{\frac{q}{p+q}}\left(\int_{a}^{x} \frac{1}{r^{\frac{1}{p+q-1}}(t)} \Delta t\right)^{\frac{p(p+q-1)}{p+q}} z^{\frac{p}{p+q}}(x)\left(z^{\Delta}(x)\right)^{\frac{q}{p+q}} \Delta x
\end{aligned}
$$




$$
\begin{aligned}
& +2^{3 p-2}|y(a)|^{p} \int_{a}^{\tau} s(x)\left(\frac{z^{\Delta}(x)}{r(x)}\right)^{\frac{q}{p+q}} \Delta x+2^{p-1} \int_{a}^{\tau}\left(\frac{\mu^{p}(x) s(x)}{r(x)}\right) z^{\Delta}(x) \Delta x \\
\leq & 2^{3 p-2} \int_{a}^{\tau} s(x)\left(\frac{1}{r(x)}\right)^{\frac{q}{p+q}}\left(\int_{a}^{x} \frac{1}{r^{\frac{1}{p+q-1}}(t)} \Delta t\right)^{\frac{p(p+q-1)}{p+q}} z^{\frac{p}{p+q}}(x)\left(z^{\Delta}(x)\right)^{\frac{q}{p+q}} \Delta x \\
& +2^{3 p-2}|y(a)|^{p} \int_{a}^{\tau} s(x)\left(\frac{z^{\Delta}(x)}{r(x)}\right)^{\frac{q}{p+q}} \Delta x+2^{p-1} \max _{a \leq x \leq \tau}\left(\frac{\mu^{p}(x) s(x)}{r(x)}\right) \int_{a}^{\tau} z^{\Delta}(x) \Delta x .
\end{aligned}
$$

By applying Hölder inequality (3.3) with $(p+q) / p$ and $(p+q) / q$ on the right side of integral of the above inequality, we have

$$
\begin{aligned}
& \int_{a}^{\tau} s(x)\left|y(x)+y^{\sigma}(x)\right|^{p}\left|y^{\Delta}(x)\right|^{q} \Delta x \\
& \leq 2^{3 p-2}\left[\int_{a}^{\tau} s^{\frac{p+q}{p}}(x)\left(\frac{1}{r(x)}\right)^{\frac{q}{p}}\left(\int_{a}^{x} \frac{1}{r^{\frac{1}{p+q-1}}(t)} \Delta t\right)^{p+q-1} \Delta x\right]^{\frac{p}{p+q}} \\
& \quad \times\left[\int_{a}^{\tau} z^{\frac{p}{q}}(x) z^{\Delta}(x) \Delta x\right]^{\frac{q}{p+q}} \\
& +2^{3 p-2}|y(a)|^{p} \int_{a}^{\tau} s(x)\left(\frac{z^{\Delta}(x)}{r(x)}\right)^{\frac{q}{p+q}} \Delta x \\
& \quad+2^{p-1} \max _{a \leq x \leq \tau}\left(\frac{\mu^{p}(x) s(x)}{r(x)}\right) \int_{a}^{\tau} z^{\Delta}(x) \Delta x .
\end{aligned}
$$

From (3.1), we obtain

$$
\left[z^{(p+q) / q}\right]^{\Delta}(x)=\frac{p+q}{q} z^{p / q}(c) z^{\Delta}(x), \quad c \in[x, \sigma(x)] .
$$

Since $z^{\Delta}(x) \geq 0$ and $x \leq c$, we get

$$
\left[z^{\frac{p+q}{q}}\right]^{\Delta}(x)=\frac{p+q}{q} z^{p / q}(c) z^{\Delta}(x) \geq \frac{p+q}{q} z^{p / q}(x) z^{\Delta}(x) .
$$

Substituting (3.19) into (3.18) and since $z(a)=0$, we have

$$
\begin{aligned}
\int_{a}^{\tau} s(x)\left|y(x)+y^{\sigma}(x)\right|^{p}\left|y^{\Delta}(x)\right|^{q} \Delta x \\
\leq 2^{3 p-2}\left(\frac{q}{p+q}\right)^{\frac{q}{p+q}}\left[\int _ { a } ^ { \tau } s ^ { \frac { p + q } { p } } ( x ) ( \frac { 1 } { r ( x ) } ) ^ { \frac { q } { p } } \left(\int_{a}^{x} \frac{1}{\left.\left.r^{\frac{1}{p+q-1}(t)} \Delta t\right)^{p+q-1} \Delta x\right]^{\frac{p}{p+q}}}\right.\right. \\
\quad \times\left[\int_{a}^{\tau}\left(z^{\frac{p+q}{q}}\right)^{\Delta}(x) \Delta x\right]^{\frac{q}{p+q}}+2^{3 p-2}|y(a)|^{p} \int_{a}^{\tau} s(x)\left(\frac{z^{\Delta}(x)}{r(x)}\right)^{\frac{q}{p+q}} \Delta x \\
\quad+2^{p-1} \max _{a \leq x \leq \tau}\left(\frac{\mu^{p}(x) s(x)}{r(x)}\right) \int_{a}^{\tau} z^{\Delta}(x) \Delta x \\
=2^{3 p-2}\left(\frac{q}{p+q}\right)^{\frac{q}{p+q}}\left[\int_{a}^{\tau} s^{\frac{p+q}{p}}(x)\left(\frac{1}{r(x)}\right)^{\frac{q}{p}}\left(\int_{a}^{x} \frac{1}{r^{\frac{1}{p+q-1}}(t)} \Delta t\right)^{p+q-1} \Delta x\right]^{\frac{p}{p+q}} z(\tau) \\
\quad+2^{3 p-2}|y(a)|^{p} \int_{a}^{\tau} s(x)\left(\frac{z^{\Delta}(x)}{r(x)}\right)^{\frac{q}{p+q}} \Delta x+2^{p-1} \frac{\max }{a \leq x \leq \tau}\left(\frac{\mu^{p}(x) s(x)}{r(x)}\right) z(\tau) .
\end{aligned}
$$


The above inequality, (3.15) and (3.16) imply that

$$
\begin{aligned}
& \int_{a}^{\tau} s(x)\left|y(x)+y^{\sigma}(x)\right|^{p}\left|y^{\Delta}(x)\right|^{q} \Delta x \\
& \quad \leq K_{5}(a, \tau, p, q) \int_{a}^{\tau} r(x)\left|y^{\Delta}(x)\right|^{p+q} \Delta x+2^{3 p-2}|y(a)|^{p} \int_{a}^{\tau} s(x)\left|y^{\Delta}(x)\right|^{q} \Delta x
\end{aligned}
$$

which is the desired inequality (3.6).

(b) The proof follows from (a) by setting $r=s$.

(c) It is noted from the chain rule on time scales (3.2) that

$$
\begin{aligned}
\left((t-a)^{p+q}\right)^{\Delta} & =(p+q) \int_{0}^{1}[h(\sigma(t)-a)+(1-h)(t-a)]^{p+q-1} d h \\
& \geq(p+q) \int_{0}^{1}[h(t-a)+(1-h)(t-a)]^{p+q-1} d h \\
& =(p+q)(t-a)^{p+q-1},
\end{aligned}
$$

so that

$$
\int_{a}^{\tau}(x-a)^{p+q-1} \Delta x \leq \int_{a}^{\tau} \frac{1}{(p+q)}\left((x-a)^{p+q}\right)^{\Delta} \Delta x=\frac{(\tau-a)^{p+q}}{(p+q)} .
$$

From (3.7) and (3.8) (by taking $r(t)=1$ ) and using (3.20), we get

$$
\begin{aligned}
& \int_{a}^{\tau}\left|y(x)+y^{\sigma}(x)\right|^{p}\left|y^{\Delta}(x)\right|^{q} \Delta x \\
& \leq {\left[2^{3 p-2}\left(\frac{q}{p+q}\right)^{\frac{q}{p+q}}\left(\int_{a}^{\tau}(x-a)^{(p+q-1)} \Delta x\right)^{\frac{p}{p+q}}+2^{p-1} \max _{a \leq x \leq \tau}\left(\mu^{p}(x)\right)\right] } \\
& \times \int_{a}^{\tau} r(x)\left|y^{\Delta}(x)\right|^{p+q} \Delta x+2^{p-1} \max _{a \leq x \leq \tau}\left(\mu^{p}(x)\right) \\
& \leq {\left[2^{3 p-2}\left(\frac{q}{p+q}\right)^{\frac{q}{p+q}}\left(\frac{(\tau-a)^{p+q}}{(p+q)}\right)^{\frac{p}{p+q}}+2^{p-1} \max _{a \leq x \leq \tau}\left(\mu^{p}(x)\right)\right] \int_{a}^{\tau} r(x)\left|y^{\Delta}(x)\right|^{p+q} \Delta x } \\
&+2^{p-1} \max _{a \leq x \leq \tau}\left(\mu^{p}(x)\right) \\
&= {\left[2^{3 p-2} \frac{q^{\frac{q}{p+q}}}{p+q}(\tau-a)^{p}+2^{p-1} \max _{a \leq x \leq \tau}\left(\mu^{p}(x)\right)\right] \int_{a}^{\tau} r(x)\left|y^{\Delta}(x)\right|^{p+q} \Delta x } \\
&+2^{p-1} \max _{a \leq x \leq \tau}\left(\mu^{p}(x)\right),
\end{aligned}
$$

which is the desired inequality (3.9). This completes the proof.

Based on Theorem 3.4, we obtain the following result by replacing $[a, \tau]_{\mathbb{T}}$ by $[\tau, b]_{\mathbb{T}}$ and $|y(x)|=\int_{x}^{b}\left|y^{\Delta}(t)\right| \Delta t+|y(b)|$.

Theorem 3.5 Let (A1)(ii), (A2)(i), (A3)(ii) and (A5) hold. Then

$$
\int_{\tau}^{b} s(x)\left|y(x)+y^{\sigma}(x)\right|^{p}\left|y^{\Delta}(x)\right|^{q} \Delta x
$$




$$
\leq K_{8}(\tau, b, p, q) \int_{\tau}^{b} r(x)\left|y^{\Delta}(x)\right|^{p+q} \Delta x+2^{3 p-2}|y(b)|^{p} \int_{\tau}^{b} s(x)\left|y^{\Delta}(x)\right|^{q} \Delta x,
$$

where

$$
\begin{aligned}
K_{8}(\tau, b, p, q)= & 2^{3 p-2}\left(\frac{q}{p+q}\right)^{\frac{q}{p+q}}\left[\int_{\tau}^{b} s^{\frac{p+q}{p}}(x)\left(\frac{1}{r(x)}\right)^{\frac{q}{p}}\left(\int_{x}^{b} \frac{1}{r^{\frac{1}{p+q-1}}(t)} \Delta t\right)^{p+q-1} \Delta x\right]^{\frac{p}{p+q}} \\
& +2^{p-1} \max _{\tau \leq x \leq b}\left(\frac{\mu^{p}(x) s(x)}{r(x)}\right) .
\end{aligned}
$$

Let $K_{2}^{\star}(p, q)=K_{7}(a, \tau, p, q)=K_{8}(\tau, b, p, q)<\infty$ such that $K_{7}(a, \tau, p, q)$ and $K_{8}(\tau, b, p, q)$ are given in Theorems 3.4 and 3.5 and $\tau$ is the unique solution of the equation $K_{7}(a, \tau, p, q)=$ $K_{8}(\tau, b, p, q)$. Therefore,

$$
\begin{aligned}
& \int_{a}^{b} s(x)\left|y(x)+y^{\sigma}(x)\right|^{p}\left|y^{\Delta}(x)\right|^{q} \Delta x \\
& \quad=\int_{a}^{\tau} s(x)\left|y(x)+y^{\sigma}(x)\right|^{p}\left|y^{\Delta}(x)\right|^{q} \Delta x+\int_{\tau}^{b} s(x)\left|y(x)+y^{\sigma}(x)\right|^{p}\left|y^{\Delta}(x)\right|^{q} \Delta x .
\end{aligned}
$$

So combining Theorems 3.4 and 3.5 gives the following result.

Theorem 3.6 Let (A1)(iii), (A2)(i), (A3)(iii) and (A6) be fulfilled.

(a) Then

$$
\begin{aligned}
& \int_{a}^{b} s(x)\left|y(x)+y^{\sigma}(x)\right|^{p}\left|y^{\Delta}(x)\right|^{q} \Delta x \\
& \quad \leq K_{2}^{\star}(p, q) \int_{a}^{b} r(x)\left|y^{\Delta}(x)\right|^{p+q} \Delta x \\
& \quad+2^{3 p-2}\left(|y(a)|^{p}+|y(b)|^{p}\right) \int_{a}^{b} s(x)\left|y^{\Delta}(x)\right|^{q} \Delta x .
\end{aligned}
$$

(b) By applying (3.9) for $[a, \tau]$ and $[\tau, b]$ and choosing $\tau=\frac{a+b}{2} \in \mathbb{T}$, therefore

$$
\begin{aligned}
& \int_{a}^{b}\left|y(x)+y^{\sigma}(x)\right|^{p}\left|y^{\Delta}(x)\right|^{q} \Delta x \\
& \leq K_{9}(a, b, p, q) \int_{a}^{b}\left|y^{\Delta}(x)\right|^{p+q} \Delta x \\
& \quad+2^{3 p-2}\left(|y(a)|^{p}+|y(b)|^{p}\right) \int_{a}^{b}\left|y^{\Delta}(x)\right|^{q} \Delta x,
\end{aligned}
$$

where

$$
K_{9}(a, b, p, q)=\left(2^{3 p-2} \frac{q^{\frac{q}{p+q}}}{p+q}\left(\frac{b-a}{2}\right)^{p}+2^{p-1} \max _{a \leq x \leq \tau}\left(\mu^{p}(x)\right)\right) .
$$

(c) Setting $p=q=1$ in (3.23), hence

$$
\int_{a}^{b}\left|y(x)+y^{\sigma}(x)\right|\left|y^{\Delta}(x)\right| \Delta x
$$




$$
\begin{aligned}
\leq & \left(\frac{b-a}{2}+\max _{a \leq x \leq b} \mu(x)\right) \int_{a}^{b}\left|y^{\Delta}(x)\right|^{2} \Delta x \\
& +2(|y(a)|+|y(b)|) \int_{a}^{b}\left|y^{\Delta}(x)\right| \Delta x .
\end{aligned}
$$

Listed below are some remarks on particular cases of Theorem 3.4, Theorem 3.5 and Theorem 3.6:

Remark 3.7 If we take $y(a)=0$, the inequality (3.6) reduces to the inequality (1.7).

Remark 3.8 If we take $y(a)=0$ and $r=s$, the inequality (3.7) reduces to the inequality [6, (3.3.16), page 126].

Remark 3.9 The inequality (3.9) changes to the inequality [6, (3.3.19), page 126] by putting $y(a)=0$ and $r=s=1$.

Remark 3.10 If we take $y(b)=0$, the inequality (3.21) reduces to the inequality (1.8).

Remark 3.11 If we take $y(a)=0$ and $y(b)=0$, the inequality (3.22) reduces to the inequality (1.9).

Remark 3.12 If we take $y(a)=0$ and $y(b)=0, r=s=1$ and choose $\tau=\frac{a+b}{2} \in \mathbb{T}$, the inequality (3.23) reduces to the inequality [6, (3.3.20), page 126].

Remark 3.13 If we take $y(a)=0$ and $y(b)=0$ the inequality (3.24) reduces to the inequality [6, (3.3.21), page 127].

Now, we give some integral and discrete inequalities as special cases from Theorems $3.4,3.5$ and 3.6 , respectively:

Corollary 3.14 When $\mathbb{T}=\mathbb{R}$ in Theorem 3.4, and using Eqs. (2.4), the inequality (3.6) reduces to

$$
\begin{aligned}
& \int_{a}^{\tau} s(x)|y(x)|^{p}\left|y^{\prime}(x)\right|^{q} d x \\
& \quad \leq K_{10}(a, \tau, p, q) \int_{a}^{\tau} r(x)\left|y^{\prime}(x)\right|^{p+q} d x+2^{2(p-1)}|y(a)|^{p} \int_{a}^{\tau} s(x)\left|y^{\prime}(x)\right|^{q} d x,
\end{aligned}
$$

where

$$
\begin{aligned}
& K_{10}(a, \tau, p, q) \\
& \quad=2^{2(p-1)}\left(\frac{q}{p+q}\right)^{\frac{q}{p+q}}\left[\int_{a}^{\tau} s^{\frac{p+q}{p}}(x)\left(\frac{1}{r(x)}\right)^{\frac{q}{p}}\left(\int_{a}^{x} \frac{1}{r^{\frac{1}{p+q-1}}(t)} d t\right)^{p+q-1} d x\right]^{\frac{p}{p+q}} .
\end{aligned}
$$

Corollary 3.15 When $\mathbb{T}=\mathbb{R}$, in Theorem 3.5, and using Eqs. (2.4), the inequality (3.21) reduces to

$$
\int_{\tau}^{b} s(x)|y(x)|^{p}\left|y^{\prime}(x)\right|^{q} d x
$$




$$
\leq K_{11}(\tau, b, p, q) \int_{\tau}^{b} r(x)\left|y^{\prime}(x)\right|^{p+q} d x+2^{2(p-1)}|y(b)|^{p} \int_{\tau}^{b} s(x)\left|y^{\prime}(x)\right|^{q} d x
$$

where

$$
\begin{aligned}
& K_{11}(\tau, b, p, q) \\
& \quad=2^{2(p-1)}\left(\frac{q}{p+q}\right)^{\frac{q}{p+q}}\left[\int_{\tau}^{b} s^{\frac{p+q}{p}}(x)\left(\frac{1}{r(x)}\right)^{\frac{q}{p}}\left(\int_{x}^{b} \frac{1}{r^{\frac{1}{p+q-1}}(t)} d t\right)^{p+q-1} d x\right]^{\frac{p}{p+q}} .
\end{aligned}
$$

Corollary 3.16 When $\mathbb{T}=\mathbb{R}$, in Theorem 3.6, and using Eqs. (2.4), the inequality (3.22) reduces to

$$
\begin{aligned}
& \int_{a}^{b} s(x)|y(x)|^{p}\left|y^{\prime}(x)\right|^{q} d x \\
& \quad \leq K_{3}^{\star}(p, q) \int_{a}^{b} r(x)\left|y^{\prime}(x)\right|^{p+q} d x+2^{2(p-1)}(|y(a)|+|y(b)|)^{p} \int_{a}^{b} s(x)\left|y^{\prime}(x)\right|^{q} d x,
\end{aligned}
$$

where $K_{3}^{\star}(p, q)=K_{10}(a, \tau, p, q)=K_{11}(\tau, b, p, q)<\infty$ such that $K_{10}(a, \tau, p, q)$ and $K_{11}(\tau, b, p, q)$ are given in Corollaries 3.14 and 3.15 and $\tau$ is the unique solution of the equation $K_{10}(a, \tau, p, q)=K_{11}(\tau, b, p, q)$.

Corollary 3.17 If $\mathbb{T}=\mathbb{N}$ in Theorem 3.4 and $(A 2)(i),(A 7),(A 8)(i)$ are satisfied, and using Eqs. (2.5), then

$$
\begin{aligned}
& \sum_{n=a}^{N-1} s(n)|y(n)+y(n+1)|^{p}|\Delta y(n)|^{q} \\
& \quad \leq K_{12}(a, \tau, p, q) \sum_{n=a}^{N-1} r(n)|\Delta y(n)|^{p+q}+2^{3 p-2}|y(a)|^{p} \sum_{n=a}^{N-1} s(n)|\Delta y(n)|^{q},
\end{aligned}
$$

where

$$
\begin{aligned}
K_{12}(a, \tau, p, q)= & 2^{3 p-2}\left(\frac{q}{p+q}\right)^{\frac{q}{p+q}}\left[\sum_{n=a}^{N-1} s^{\frac{p+q}{p}}(n)\left(\frac{1}{r(n)}\right)^{\frac{q}{p}}\left(\sum_{n=a}^{N-1} \frac{1}{r^{\frac{1}{p+q-1}}(n)}\right)^{p+q-1}\right]^{\frac{p}{p+q}} \\
& +2^{p-1} \max _{a \leq x \leq \tau}\left(\frac{s(n)}{r(n)}\right) .
\end{aligned}
$$

Corollary 3.18 If $\mathbb{T}=\mathbb{N}$ in Theorem 3.5 and $(A 2)(i)$, (A7), (A8)(ii) hold, and using Eqs. (2.5), then

$$
\begin{aligned}
& \sum_{n=N}^{b-1} s(n)|y(n)+y(n+1)|^{p}|\Delta y(n)|^{q} \\
& \quad \leq K_{13}(\tau, b, p, q) \sum_{n=N}^{b-1} r(n)|\Delta y(n)|^{p+q}+2^{3 p-2}|y(b)|^{p} \sum_{n=N}^{b-1} s(n)|\Delta y(n)|^{q}
\end{aligned}
$$


where

$$
\begin{aligned}
K_{13}(\tau, b, p, q)= & 2^{3 p-2}\left(\frac{q}{p+q}\right)^{\frac{q}{p+q}}\left[\sum_{n=N}^{b-1} s^{\frac{p+q}{p}}(n)\left(\frac{1}{r(n)}\right)^{\frac{q}{p}}\left(\sum_{n=N}^{b-1} \frac{1}{r^{\frac{1}{p+q-1}}(n)}\right)^{p+q-1}\right]^{\frac{p}{p+q}} \\
& +2^{p-1} \max _{x \leq b \leq \tau}\left(\frac{s(n)}{r(n)}\right) .
\end{aligned}
$$

Corollary 3.19 If $\mathbb{T}=\mathbb{N}$ in Theorem 3.6, and $(A 2)(i),(A 7)$, (A8)(iii) are satisfied, and using Eqs. (2.5), then

$$
\begin{aligned}
& \sum_{n=a}^{b-1} s(n)|y(n)+y(n+1)|^{p}|\Delta y(n)|^{q} \\
& \quad \leq K_{4}^{\star}(p, q) \sum_{n=a}^{b-1} r(n)|\Delta y(n)|^{p+q}+2^{3 p-2}(|y(a)|+|y(b)|)^{p} \sum_{n=a}^{b-1} s(n)|\Delta y(n)|^{q},
\end{aligned}
$$

where $K_{4}^{\star}(p, q)=K_{12}(a, \tau, p, q)=K_{13}(\tau, b, p, q)<\infty$ such that $K_{12}(a, \tau, p, q)$ and $K_{13}(\tau, b, p, q)$ are given in Corollaries 3.17 and 3.18 and $\tau$ is the unique solution of the equation $K_{12}(a, \tau, p, q)=K_{13}(\tau, b, p, q)$.

Now we study the case of some weighted dynamic Opial inequalities on time scales of the type

$$
\begin{aligned}
& \int_{a}^{\tau} s(x)\left|y(x)+y^{\sigma}(x)\right|^{p}\left|y^{\Delta}(x)\right|^{q} \Delta x \\
& \quad \leq K_{1}(a, \tau, p, q) \int_{a}^{\tau} r(x)\left|y^{\Delta}(x)\right|^{p+q} \Delta x+2^{3 p-2}|y(a)|^{p} \int_{a}^{\tau} s(x)\left|y^{\Delta}(x)\right|^{q} \Delta x,
\end{aligned}
$$

where $p, q$ be positive real numbers such that $p \leq 1, p+q>1$.

Our next results, which will be proved by using inequality (3.5), generalize the inequalities (1.10), (1.11) and (1.12).

Theorem 3.20 Assume $(A 1)(i),(A 2)((i i),(i i i)),(A 3)(i)$ and $(A 4)$ are fulfilled.

(a) Then

$$
\begin{aligned}
& \int_{a}^{\tau} s(x)\left|y(x)+y^{\sigma}(x)\right|^{p}\left|y^{\Delta}(x)\right|^{q} \Delta x \\
& \quad \leq K_{14}(a, \tau, p, q) \int_{a}^{\tau} r(x)\left|y^{\Delta}(x)\right|^{p+q} \Delta x+2^{p}|y(a)|^{p} \int_{a}^{\tau} s(x)\left|y^{\Delta}(x)\right|^{q} \Delta x,
\end{aligned}
$$

where

$$
\begin{aligned}
& K_{14}(a, \tau, p, q) \\
& =2^{p}\left(\frac{q}{p+q}\right)^{\frac{q}{p+q}}\left[\int_{a}^{\tau} s^{\frac{p+q}{p}}(x)\left(\frac{1}{r(x)}\right)^{\frac{q}{p}}\left(\int_{a}^{x} \frac{1}{r^{\frac{1}{p+q-1}}(t)} \Delta t\right)^{p+q-1} \Delta x\right]^{\frac{p}{p+q}} \\
& \quad+\max _{a \leq x \leq \tau}\left(\frac{\mu^{p}(x) s(x)}{r(x)}\right) .
\end{aligned}
$$


(b) For $r=s$, we obtain

$$
\begin{aligned}
& \int_{a}^{\tau} r(x)\left|y(x)+y^{\sigma}(x)\right|^{p}\left|y^{\Delta}(x)\right|^{q} \Delta x \\
& \quad \leq K_{15}(a, \tau, p, q) \int_{a}^{\tau} r(x)\left|y^{\Delta}(x)\right|^{p+q} \Delta+2^{p}|y(a)|^{p} \int_{a}^{\tau} r(x)\left|y^{\Delta}(x)\right|^{q} \Delta x,
\end{aligned}
$$

where

$$
\begin{aligned}
& K_{15}(a, \tau, p, q)=2^{p}\left(\frac{q}{p+q}\right)^{\frac{q}{p+q}}\left[\int_{a}^{\tau} r(x)\left(\int_{a}^{x} \frac{1}{r^{\frac{1}{p+q-1}}(t)} \Delta t\right)^{p+q-1} \Delta x\right]^{\frac{p}{p+q}} \\
& +\max _{a \leq x \leq \tau}\left(\mu^{p}(x)\right) \text {. }
\end{aligned}
$$

(c) Setting $r=1$ in (3.26) and (3.27), then

$$
\begin{aligned}
& \int_{a}^{\tau}\left|y(x)+y^{\sigma}(x)\right|^{p}\left|y^{\Delta}(x)\right|^{q} \Delta x \\
& \quad \leq K_{16}(a, \tau, p, q) \int_{a}^{\tau}\left|y^{\Delta}(x)\right|^{p+q} \Delta+2^{p}|y(a)|^{p} \int_{a}^{\tau}\left|y^{\Delta}(x)\right|^{q} \Delta x,
\end{aligned}
$$

where

$$
K_{16}(a, \tau, p, q)=\left(2^{p} \frac{q^{\frac{q}{p+q}}}{p+q}(\tau-a)^{p}+\max _{a \leq x \leq \tau}\left(\mu^{p}(x)\right)\right) .
$$

Proof (a) Since $y^{\Delta}(t)$ does not change sign in $(a, \tau)_{\mathbb{T}}$, we have

$$
|y(x)|-|y(a)| \leq|y(x)-y(a)|=\left|\int_{a}^{x} y^{\Delta}(t) \Delta t\right| \leq \int_{a}^{x}\left|y^{\Delta}(t)\right| \Delta t .
$$

From (3.29), we get

$$
|y(x)| \leq \int_{a}^{x}\left|y^{\Delta}(t)\right| \Delta t+|y(a)|=\int_{a}^{x} \frac{1}{r^{\frac{1}{p+q}}(t)} r^{\frac{1}{p+q}}(t)\left|y^{\Delta}(t)\right| \Delta t+|y(a)| .
$$

Now, since $r$ is nonnegative on $(a, \tau)_{\mathbb{T}}$, then it follows from the Hölder inequality (3.3) with indices $\frac{p+q}{p+q-1}$ and $p+q$, and with

$$
f(t)=\frac{1}{r^{\frac{1}{p+q}}(t)}, \quad g(t)=r^{\frac{1}{p+q}}(t)\left|y^{\Delta}(t)\right|,
$$

that

$$
|y(x)| \leq\left(\int_{a}^{x} \frac{1}{r^{\frac{1}{p+q-1}}(t)} \Delta t\right)^{\frac{p+q-1}{p+q}}\left(\int_{a}^{x} r(t)\left|y^{\Delta}(t)\right|^{p+q} \Delta t\right)^{\frac{1}{p+q}}+|y(a)| .
$$

Since $p \leq 1$, by taking the power $p$ for both sides of (3.30), we have

$$
|y(x)|^{p} \leq\left(\left(\int_{a}^{x} \frac{1}{r^{\frac{1}{p+q-1}}(t)} \Delta t\right)^{\frac{p+q-1}{p+q}}\left(\int_{a}^{x} r(t)\left|y^{\Delta}(t)\right|^{p+q} \Delta t\right)^{\frac{1}{p+q}}+|y(a)|\right)^{p} .
$$


Applying the inequality (3.5) on the right-hand side of (3.31), we deduce

$$
|y(x)|^{p} \leq\left(\int_{a}^{x} \frac{1}{r^{\frac{1}{p+q-1}}(t)} \Delta t\right)^{\frac{p(p+q-1)}{p+q}}\left(\int_{a}^{x} r(t)\left|y^{\Delta}(t)\right|^{p+q} \Delta t\right)^{\frac{p}{p+q}}+|y(a)|^{p} .
$$

Since $y^{\sigma}=y+\mu y^{\Delta}$, we have

$$
y(x)+y^{\sigma}(x)=2 y(x)+\mu y^{\Delta}(x) .
$$

Since $p \leq 1$, by taking the power $p$ of both sides of (3.32) and applying again the inequality (3.5), we deduce

$$
\left|y(x)+y^{\sigma}(x)\right|^{p}=\left|2 y(x)+\mu y^{\Delta}(x)\right|^{p} \leq 2^{p}|y(x)|^{p}+\mu^{p}(x)\left|y^{\Delta}(x)\right|^{p} .
$$

Setting

$$
z(x):=\int_{a}^{x} r(t)\left|y^{\Delta}(t)\right|^{p+q} \Delta t
$$

using the fact that $z(a)=0$, and

$$
z^{\Delta}(x)=r(x)\left|y^{\Delta}(x)\right|^{p+q}>0 .
$$

This implies

$$
\left|y^{\Delta}(x)\right|^{p+q}=\frac{z^{\Delta}(x)}{r(x)} \quad \text { and } \quad\left|y^{\Delta}(x)\right|^{q}=\left(\frac{z^{\Delta}(x)}{r(x)}\right)^{\frac{q}{p+q}} .
$$

From (3.33) and (3.36), since $s$ is nonnegative on $(a, \tau)_{\mathbb{T}}$, we have

$$
\begin{aligned}
& s(x)\left|y(x)+y^{\sigma}(x)\right|^{p}\left|y^{\Delta}(x)\right|^{q} \\
& \leq 2^{p} s(x)|y(x)|^{p}\left|y^{\Delta}(x)\right|^{q}+\mu^{p}(x) s(x)\left|y^{\Delta}\right|^{p+q} \\
& \leq 2^{p} s(x)\left(\frac{1}{r(x)}\right)^{\frac{q}{p+q}}\left(\int_{a}^{x} \frac{1}{r^{\frac{1}{p+q-1}}(t)} \Delta t\right)^{\frac{p(p+q-1)}{p+q}} \\
& \quad \times z^{\frac{p}{p+q}}(x)\left(z^{\Delta}(x)\right)^{\frac{q}{p+q}}+2^{p} s(x)|y(a)|^{p}\left(\frac{z^{\Delta}(x)}{r(x)}\right)^{\frac{q}{p+q}} \\
&+\mu^{p}(x) s(x) \frac{z^{\Delta}(x)}{r(x)} .
\end{aligned}
$$

Integrating the above inequality from $a$ to $\tau$, we get

$$
\begin{aligned}
& \int_{a}^{\tau} s(x)\left|y(x)+y^{\sigma}(x)\right|^{p}\left|y^{\Delta}(x)\right|^{q} \Delta x \\
& \quad \leq 2^{p} \int_{a}^{\tau} s(x)\left(\frac{1}{r(x)}\right)^{\frac{q}{p+q}}\left(\int_{a}^{x} \frac{1}{r^{\frac{1}{p+q-1}}(t)} \Delta t\right)^{\frac{p(p+q-1)}{p+q}} z^{\frac{p}{p+q}}(x)\left(z^{\Delta}(x)\right)^{\frac{q}{p+q}} \Delta x
\end{aligned}
$$




$$
\begin{aligned}
& +2^{p}|y(a)|^{p} \int_{a}^{\tau} s(x)\left(\frac{z^{\Delta}(x)}{r(x)}\right)^{\frac{q}{p+q}} \Delta x+\int_{a}^{\tau}\left(\frac{\mu^{p}(x) s(x)}{r(x)}\right) z^{\Delta}(x) \Delta x \\
\leq & 2^{p} \int_{a}^{\tau} s(x)\left(\frac{1}{r(x)}\right)^{\frac{q}{p+q}}\left(\int_{a}^{x} \frac{1}{r^{\frac{1}{p+q-1}}(t)} \Delta t\right)^{\frac{p(p+q-1)}{p+q}} z^{\frac{p}{p+q}}(x)\left(z^{\Delta}(x)\right)^{\frac{q}{p+q}} \Delta x \\
& +2^{p}|y(a)|^{p} \int_{a}^{\tau} s(x)\left(\frac{z^{\Delta}(x)}{r(x)}\right)^{\frac{q}{p+q}} \Delta x+\max _{a \leq x \leq \tau}\left(\frac{\mu^{p}(x) s(x)}{r(x)}\right) \int_{a}^{\tau} z^{\Delta}(x) \Delta x .
\end{aligned}
$$

Applying the Hölder inequality (3.3), with indices $(p+q) / p$ and $(p+q) / q$ on the first integral of the right-hand side of the above inequality, we have

$$
\begin{aligned}
& \int_{a}^{\tau} s(x)\left|y(x)+y^{\sigma}(x)\right|^{p}\left|y^{\Delta}(x)\right|^{q} \Delta x \\
& \leq 2^{p}\left[\int_{a}^{\tau} s^{\frac{p+q}{p}}(x)\left(\frac{1}{r(x)}\right)^{\frac{q}{p}}\left(\int_{a}^{x} \frac{1}{r^{\frac{1}{p+q-1}}(t)} \Delta t\right)^{p+q-1} \Delta x\right]^{\frac{p}{p+q}}\left[\int_{a}^{\tau} z^{\frac{p}{q}}(x) z^{\Delta}(x) \Delta x\right]^{\frac{q}{p+q}} \\
& \quad+2^{p}|y(a)|^{p} \int_{a}^{\tau} s(x)\left(\frac{z^{\Delta}(x)}{r(x)}\right)^{\frac{q}{p+q}} \Delta x+\max _{a \leq x \leq \tau}\left(\frac{\mu^{p}(x) s(x)}{r(x)}\right) \int_{a}^{\tau} z^{\Delta}(x) \Delta x
\end{aligned}
$$

From the chain rule (3.1), we obtain

$$
\left[z^{(p+q) / q}\right]^{\Delta}(x)=\frac{p+q}{q} z^{p / q}(c) z^{\Delta}(x), \quad c \in[x, \sigma(x)] .
$$

Since $z^{\Delta}(x) \geq 0$ and $x \leq c$, we get

$$
\left[z^{\frac{p+q}{q}}\right]^{\Delta}(x)=\frac{p+q}{q} z^{p / q}(c) z^{\Delta}(x) \geq \frac{p+q}{q} z^{p / q}(x) z^{\Delta}(x) .
$$

Substituting (3.38) into (3.37) and by $z(a)=0$, we have

$$
\begin{aligned}
\int_{a}^{\tau} s(x)\left|y(x)+y^{\sigma}(x)\right|^{p}\left|y^{\Delta}(x)\right|^{q} \Delta x \\
\leq 2^{p}\left(\frac{q}{p+q}\right)^{\frac{q}{p+q}}\left[\int_{a}^{\tau} s^{\frac{p+q}{p}}(x)\left(\frac{1}{r(x)}\right)^{\frac{q}{p}}\left(\int_{a}^{x} \frac{1}{r^{\frac{1}{p+q-1}}(t)} \Delta t\right)^{p+q-1} \Delta x\right]^{\frac{p}{p+q}} \\
\quad \times\left[\int_{a}^{\tau}\left(z^{\frac{p+q}{q}}\right)^{\Delta}(x) \Delta x\right]^{\frac{q}{p+q}}+2^{p}|y(a)|^{p} \int_{a}^{\tau} s(x)\left(\frac{z^{\Delta}(x)}{r(x)}\right)^{\frac{q}{p+q}} \Delta x \\
\quad+\max _{a \leq x \leq \tau}\left(\frac{\mu^{p}(x) s(x)}{r(x)}\right) \int_{a}^{\tau} r(x)\left|y^{\Delta}(x)\right|^{p+q} \Delta x \\
=2^{p}\left(\frac{q}{p+q}\right)^{\frac{q}{p+q}}\left[\int_{a}^{\tau} s^{\frac{p+q}{p}}(x)\left(\frac{1}{r(x)}\right)^{\frac{q}{p}}\left(\int_{a}^{x} \frac{1}{r^{\frac{1}{p+q-1}}(t)} \Delta t\right)^{p+q-1} \Delta x\right]^{\frac{p}{p+q}} z(\tau) \\
\left.\quad+2^{p}|y(a)|^{p} \int_{a}^{\tau} s(x)\left(\frac{z^{\Delta}(x)}{r(x)}\right)^{\frac{q}{p+q}} \Delta x+\max _{a \leq x \leq \tau} \frac{\mu^{p}(x) s(x)}{r(x)}\right) z(\tau) .
\end{aligned}
$$

The last inequality, (3.34) and (3.35) imply that

$$
\int_{a}^{\tau} s(x)\left|y(x)+y^{\sigma}(x)\right|^{p}\left|y^{\Delta}(x)\right|^{q} \Delta x
$$




$$
\leq K_{14}(a, \tau, p, q) \int_{a}^{\tau} r(x)\left|y^{\Delta}(x)\right|^{p+q} \Delta x+2^{p}|y(a)|^{p} \int_{a}^{\tau} s(x)\left|y^{\Delta}(x)\right|^{q} \Delta x,
$$

which is the required inequality (3.25).

The proof of (b) and (c) follows by a similar argument to the proof of (a) with suitable changes. This completes the proof.

Based on Theorem 3.20, we obtain the following result by replacing $[a, \tau]_{\mathbb{T}}$ by $[\tau, b]_{\mathbb{T}}$ and $|y(x)|=\int_{x}^{b}\left|y^{\Delta}(t)\right| \Delta t+|y(b)|$.

Theorem 3.21 Assume (A1)(ii), (A2)((ii), (iii)), (A3)(ii), and (A5) are satisfied. Then we have

$$
\begin{aligned}
& \int_{\tau}^{b} s(x)\left|y(x)+y^{\sigma}(x)\right|^{p}\left|y^{\Delta}(x)\right|^{q} \Delta x \\
& \quad \leq K_{17}(\tau, b, p, q) \int_{\tau}^{b} r(x)\left|y^{\Delta}(x)\right|^{p+q} \Delta x+2^{p}|y(b)|^{p} \int_{\tau}^{b} s(x)\left|y^{\Delta}(x)\right|^{q} \Delta x,
\end{aligned}
$$

where

$$
\begin{aligned}
K_{17}(\tau, b, p, q)= & 2^{p}\left(\frac{q}{p+q}\right)^{\frac{q}{p+q}}\left[\int_{\tau}^{b} s^{\frac{p+q}{p}}(x)\left(\frac{1}{r(x)}\right)^{\frac{q}{p}}\left(\int_{x}^{b} \frac{1}{r^{\frac{1}{p+q-1}}(t)} \Delta t\right)^{p+q-1} \Delta x\right]^{\frac{p}{p+q}} \\
& +\max _{\tau \leq x \leq b}\left(\frac{\mu^{p}(x) s(x)}{r(x)}\right) .
\end{aligned}
$$

In the following, we assume that $K_{5}^{\star}(p, q)=K_{14}(a, \tau, p, q)=K_{17}(\tau, b, p, q)<\infty$, where $K_{14}(a, \tau, p, q)$ and $K_{17}(\tau, b, p, q)$ are defined as in Theorems 3.20 and 3.21 and $\tau$ is the unique solution of the equation $K_{14}(a, \tau, p, q)=K_{17}(\tau, b, p, q)$. Therefore,

$$
\begin{aligned}
& \int_{a}^{b} s(x)\left|y(x)+y^{\sigma}(x)\right|^{p}\left|y^{\Delta}(x)\right|^{q} \Delta x \\
& \quad=\int_{a}^{\tau} s(x)\left|y(x)+y^{\sigma}(x)\right|^{p}\left|y^{\Delta}(x)\right|^{q} \Delta x+\int_{\tau}^{b} s(x)\left|y(x)+y^{\sigma}(x)\right|^{p}\left|y^{\Delta}(x)\right|^{q} \Delta x .
\end{aligned}
$$

So combining Theorems 3.20 and 3.21 gives the following result.

Theorem 3.22 Assume (A1)(iii), (A2)((ii), (iii)), (A3)(iii), and (A6) are satisfied.

(a) Then

$$
\begin{aligned}
& \int_{a}^{b} s(x)\left|y(x)+y^{\sigma}(x)\right|^{p}\left|y^{\Delta}(x)\right|^{q} \Delta x \\
& \quad \leq K_{5}^{\star}(p, q) \int_{a}^{b} r(x)\left|y^{\Delta}(x)\right|^{p+q} \Delta x+2^{p}|y(a)+y(b)|^{p} \int_{a}^{b} s(x)\left|y^{\Delta}(x)\right|^{q} \Delta x .
\end{aligned}
$$

(b) Let $\tau=\frac{a+b}{2} \in \mathbb{T}$ and apply (3.28) to $[a, \tau]$ and $[\tau, b]$. Then

$$
\int_{a}^{b}\left|y(x)+y^{\sigma}(x)\right|^{p}\left|y^{\Delta}(x)\right|^{q} \Delta x
$$




$$
\leq K_{18}(a, b, p, q) \int_{a}^{b}\left|y^{\Delta}(x)\right|^{p+q} \Delta+2^{p}\left(|y(a)|^{p}+\mid y(b)^{p}\right) \int_{a}^{b}\left|y^{\Delta}(x)\right|^{q} \Delta x
$$

where

$$
K_{18}(a, b, p, q)=\left(\frac{q^{\frac{q}{p+q}}}{p+q}(b-a)^{p}+\max _{a \leq x \leq \tau}\left(\mu^{p}(x)\right)\right) .
$$

Listed below are some remarks on particular cases of Theorem 3.20, Theorem 3.21 and Theorem 3.22:

Remark 3.23 If we take $y(a)=0$, the inequality (3.25) reduces to the inequality (1.10).

Remark 3.24 If we take $y(b)=0$, the inequality (3.39) reduces to the inequality (1.11).

Remark 3.25 If we take $y(a)=0$ and $y(b)=0$, the inequality (3.40) reduces to the inequality (1.12).

Remark 3.26 If we take $y(a)=0$ and $r=s$, the inequality (3.25) reduces to the inequality [6, (3.3.32), page 130].

Remark 3.27 If we take $y(a)=0$ and $r=s=1$, the inequality (3.25) reduces to the inequality [6, (3.3.35), page 130].

Remark 3.28 If we take $y(a)=0$ and $y(b)=0, r=s=1$ and choose $\tau=\frac{(a+b)}{2}$, the inequality (3.41) reduces to the inequality [6, (3.3.36), page 131].

Now, we give some integral and discrete inequalities as special cases from Theorems $3.20,3.21$ and 3.22 , respectively:

Corollary 3.29 When $\mathbb{T}=\mathbb{R}$ in Theorem 3.20, and using Eqs. (2.4), the inequality (3.25) reduces to

$$
\begin{aligned}
& \int_{a}^{\tau} s(x)|y(x)|^{p}\left|y^{\prime}(x)\right|^{q} d x \\
& \quad \leq K_{19}(a, \tau, p, q) \int_{a}^{\tau} r(x)\left|y^{\prime}(x)\right|^{p+q} d x+|y(a)|^{p} \int_{a}^{\tau} s(x)\left|y^{\prime}(x)\right|^{q} d x,
\end{aligned}
$$

where

$$
K_{19}(a, \tau, p, q)=\left(\frac{q}{p+q}\right)^{\frac{q}{p+q}}\left[\int_{a}^{\tau} s^{\frac{p+q}{p}}(x)\left(\frac{1}{r(x)}\right)^{\frac{q}{p}}\left(\int_{a}^{x} \frac{1}{r^{\frac{1}{p+q-1}}(t)} d t\right)^{p+q-1} d x\right]^{\frac{p}{p+q}}
$$

Corollary 3.30 When $\mathbb{T}=\mathbb{R}$ in Theorem 3.21, and using Eqs. (2.4), the inequality (3.39) reduces to

$$
\begin{aligned}
& \int_{\tau}^{b} s(x)|y(x)|^{p}\left|y^{\prime}(x)\right|^{q} d x \\
& \quad \leq K_{20}(\tau, b, p, q) \int_{\tau}^{b} r(x)\left|y^{\prime}(x)\right|^{p+q} d x+|y(b)|^{p} \int_{\tau}^{b} s(x)\left|y^{\prime}(x)\right|^{q} d x,
\end{aligned}
$$


where

$$
K_{20}(\tau, b, p, q)=\left(\frac{q}{p+q}\right)^{\frac{q}{p+q}}\left[\int_{\tau}^{b} s^{\frac{p+q}{p}}(x)\left(\frac{1}{r(x)}\right)^{\frac{q}{p}}\left(\int_{x}^{b} \frac{1}{r^{\frac{1}{p+q-1}}(t)} d t\right)^{p+q-1} d x\right]^{\frac{p}{p+q}} .
$$

Corollary 3.31 When $\mathbb{T}=\mathbb{R}$ in Theorem 3.22, and using Eqs. (2.4), the inequality (3.40) reduces to

$$
\begin{aligned}
& \int_{a}^{b} s(x)|y(x)|^{p}\left|y^{\prime}(x)\right|^{q} d x \\
& \quad \leq K_{6}^{\star}(p, q) \int_{a}^{b} r(x)\left|y^{\prime}(x)\right|^{p+q} d x+(|y(a)|+|y(b)|)^{p} \int_{a}^{b} s(x)\left|y^{\prime}(x)\right|^{q} d x,
\end{aligned}
$$

where $K_{6}^{\star}(p, q)=K_{19}(a, \tau, p, q)=K_{20}(\tau, b, p, q)<\infty$ such that $K_{19}(a, \tau, p, q)$ and $K_{20}(\tau, b, p, q)$ are given in Corollaries 3.29 and 3.30 and $\tau$ is the unique solution of the equation $K_{19}(a, \tau, p, q)=K_{20}(\tau, b, p, q)$.

Corollary 3.32 If $\mathbb{T}=\mathbb{N}$ in Theorem 3.20 , and $(A 2)(i),(A 7),(A 8)(i)$ are satisfied, and using Eqs. (2.5), then

$$
\begin{aligned}
& \sum_{n=a}^{N-1} s(n)|y(n)+y(n+1)|^{p}|\Delta y(n)|^{q} \\
& \quad \leq K_{21}(a, \tau, p, q) \sum_{n=a}^{N-1} r(n)|\Delta y(n)|^{p+q}+2^{p}|y(a)|^{p} \sum_{n=a}^{N-1} s(n)|\Delta y(n)|^{q},
\end{aligned}
$$

where

$$
\begin{aligned}
K_{21}(a, \tau, p, q)= & 2^{p}\left(\frac{q}{p+q}\right)^{\frac{q}{p+q}}\left[\sum_{n=a}^{N-1} s^{\frac{p+q}{p}}(n)\left(\frac{1}{r(n)}\right)^{\frac{q}{p}}\left(\sum_{n=a}^{N-1} \frac{1}{r^{\frac{1}{p+q-1}}(n)}\right)^{p+q-1}\right]^{\frac{p}{p+q}} \\
& +\max _{a \leq x \leq \tau}\left(\frac{s(n)}{r(n)}\right) .
\end{aligned}
$$

Corollary 3.33 If $\mathbb{T}=\mathbb{N}$ in Theorem 3.21, and (A2)(ii), (A7), (A8)(ii) are satisfied, and using Eqs. (2.5), then

$$
\begin{aligned}
& \sum_{n=N}^{b-1} s(n)|y(n)+y(n+1)|^{p}|\Delta y(n)|^{q} \\
& \quad \leq K_{22}(\tau, b, p, q) \sum_{n=N}^{b-1} r(n)|\Delta y(n)|^{p+q}+2^{p}|y(b)|^{p} \sum_{n=N}^{b-1} s(n)|\Delta y(n)|^{q},
\end{aligned}
$$

where

$$
\begin{aligned}
K_{22}(\tau, b, p, q)= & 2^{p}\left(\frac{q}{p+q}\right)^{\frac{q}{p+q}}\left[\sum_{n=N}^{b-1} s^{\frac{p+q}{p}}(n)\left(\frac{1}{r(n)}\right)^{\frac{q}{p}}\left(\sum_{n=N}^{b-1} \frac{1}{r^{\frac{1}{p+q-1}(n)}}\right)^{p+q-1}\right]^{\frac{p}{p+q}} \\
& +\max _{\tau \leq x \leq b}\left(\frac{s(n)}{r(n)}\right) .
\end{aligned}
$$


Corollary 3.34 If $\mathbb{T}=\mathbb{N}$ in Theorem 3.22 and (A2)(ii), (A7), (A8)(iii) are satisfied, and using Eqs. (2.5), then

$$
\begin{aligned}
& \sum_{n=a}^{b-1} s(n)|y(n)+y(n+1)|^{p}|\Delta y(n)|^{q} \\
& \quad \leq K_{7}^{\star}(p, q) \sum_{n=a}^{b-1} r(n)|\Delta y(n)|^{p+q}+2^{p}(|y(a)|+|y(b)|)^{p} \sum_{n=a}^{b-1} s(n)|\Delta y(n)|^{q},
\end{aligned}
$$

where $K_{7}^{\star}(p, q)=K_{21}(a, \tau, p, q)=K_{22}(\tau, b, p, q)<\infty$ such that $K_{21}(a, \tau, p, q)$ and $K_{22}(\tau, b, p, q)$ are given in Corollaries 3.32 and 3.33 and $\tau$ is the unique solution of the equation $K_{21}(a, \tau, p, q)=K_{22}(\tau, b, p, q)$.

\section{Conclusion}

In this article, we obtained some weighted dynamic inequalities of Opial-type involving integrals of powers of a function and of its derivative on time scales which not only extend some results in the literature but also improve some of them. Furthermore, we got some continuous and discrete inequalities as special cases of the obtained dynamic inequalities.

\section{Acknowledgements \\ The authors wish to express their sincere appreciation to the editor and the anonymous referees for their valuable comments and suggestions.}

Funding

Not applicable.

Competing interests

The authors declare that they have no financial and non-financial competing interests.

Authors' contributions

All authors contributed equally. All the authors read and approved the final manuscript.

\section{Author details}

'Department of Mathematics, Faculty of Science, Al-Azhar University, Cairo, Egypt. ${ }^{2}$ Department of Mathematics, Faculty of Women for (Art, Science, and Education), Ain Shams University, Cairo, Egypt. ${ }^{3}$ Department of Mathematics, Princess Nourah bint Abdulrahman University, Riyadh, Saudi Arabia.

\section{Publisher's Note}

Springer Nature remains neutral with regard to jurisdictional claims in published maps and institutional affiliations.

Received: 26 May 2019 Accepted: 4 September 2019 Published online: 11 September 2019

\section{References}

1. Abdeldaim, A., El-Deeb, A.A.: Some new retarded nonlinear integral inequalities with iterated integrals and their applications in retarded differential equations and integral equations. J. Fract. Calc. Appl. 5, 9 (2014)

2. Abdeldaim, A., El-Deeb, A.A.: On generalized of certain retarded nonlinear integral inequalities and its applications in retarded integro-differential equations. Appl. Math. Comput. 256, 375-380 (2015)

3. Abdeldaim, A., El-Deeb, A.A.: On some generalizations of certain retarded nonlinear integral inequalities with iterated integrals and an application in retarded differential equation. J. Egypt. Math. Soc. 23(3), 470-475 (2015)

4. Abdeldaim, A., El-Deeb, A.A.: On some new nonlinear retarded integral inequalities with iterated integrals and their applications in integro-differential equations. Br. J. Math. Comput. Sci. 5(4), 479-491 (2015)

5. Abdeldaim, A., El-Deeb, A.A., Agarwal, P., El-Sennary, H.A.: On some dynamic inequalities of Steffensen type on time scales. Math. Methods Appl. Sci. 41(12), 4737-4753 (2018)

6. Agarwal, R., O'Regan, D., Saker, S.: Dynamic Inequalities on Time Scales. Springer, Cham (2014)

7. Agarwal, R.P., Lakshmikantham, V.: Uniqueness and Nonuniqueness Criteria for Ordinary Differential Equations. Series in Real Analysis, vol. 6. World Scientific Publishing, Singapore (1993)

8. Agarwal, R.P., Pang, P.Y.H.: Opial Inequalities with Applications in Differential and Difference Equations, vol. 320. Kluwer Academic Publishers, Dordrecht (1995)

9. Akin-Bohner, E., Bohner, M., Akin, F.: Pachpatte inequalities on time scales. JIPAM. J. Inequal. Pure Appl. Math. 6(1), 6 (2005) 
10. Beesack, P.R.: On an integral inequality of Z. Opial. Trans. Am. Math. Soc. 104, 470-475 (1962)

11. Bohner, M., Kaymakcalan, B.: Opial inequalities on time scales. Ann. Pol. Math. 77(1), 11-20 (2001)

12. Bohner, M., Matthews, T.: The Grüss inequality on time scales. Commun. Math. Anal. 3(1), 1-8 (2007)

13. Bohner, M., Matthews, T.: Ostrowski inequalities on time scales. JPAM. J. Inequal. Pure Appl. Math. 9(1), 6 (2008)

14. Bohner, M., Peterson, A.: Dynamic Equations on Time Scales: An Introduction with Applications. Birkhauser, Boston (2001)

15. Bohner, M., Peterson, A.: Advances in Dynamic Equations on Time Scales. Birkhauser, Boston (2003)

16. Dinu, C.: Hermite-Hadamard inequality on time scales. J. Inequal. Appl. 2008, Article ID 287947 (2008)

17. El-Deeb, A.A.: On Integral Inequalities and Their Applications Lambert. LAP Lambert Academic Publishing, Saarbrücken (2017)

18. El-Deeb, A.A.: A variety of nonlinear retarded integral inequalities of Gronwall type and their applications. In: Advances in Mathematical Inequalities and Applications, pp. 143-164. Springer, Berlin (2018)

19. El-Deeb, A.A.: Some Gronwall-Bellman type inequalities on time scales for Volterra-Fredholm dynamic integral equations. J. Egypt. Math. Soc. 26(1), 1-17 (2018)

20. El-Deeb, A.A.: On some generalizations of nonlinear dynamic inequalities on time scales and their applications. (To appear)

21. El-Deeb, A.A., Ahmed, R.G.: On some explicit bounds on certain retarded nonlinear integral inequalities with applications. Adv. Inequal. Appl. 2016, Article ID 15 (2016)

22. El-Deeb, A.A., Ahmed, R.G.: On some generalizations of certain nonlinear retarded integral inequalities for Volterra-Fredholm integral equations and their applications in delay differential equations. J. Egypt. Math. Soc. 25(3), 279-285 (2017)

23. El-Deeb, A.A., Cheung, W.-S.: A variety of dynamic inequalities on time scales with retardation. J. Nonlinear Sci. Appl. $11(10), 1185-1206(2018)$

24. El-Deeb, A.A., El-Sennary, H.A., Khan, Z.A.: Some Steffensen-type dynamic inequalities on time scales. Adv. Differ. Equ. 2019, 246 (2019)

25. El-Deeb, A.A., Elsennary, H.A., Cheung, W.-S.: Some reverse Hölder inequalities with Specht's ratio on time scales. J. Nonlinear Sci. Appl. 11(4), 444-455 (2018)

26. El-Deeb, A.A., Elsennary, H.A., Nwaeze, E.R.: Generalized weighted Ostrowski, trapezoid and Grüss type inequalities on time scales. Fasc. Math. 60, 123-144 (2018)

27. El-Deeb, A.A., Xu, H., Abdeldaim, A., Wang, G.: Some dynamic inequalities on time scales and their applications. Adv. Differ. Equ. 2019, 130 (2019)

28. El-Owaidy, H., Abdeldaim, A., El-Deeb, A.A.: On some new retarded nonlinear integral inequalities and their applications. Math. Sci. Lett. 3(3), 157 (2014)

29. El-Owaidy, H.M., Ragab, A.A., Eldeeb, A.A., Abuelela, W.M.K.: On some new nonlinear integral inequalities of Gronwall-Bellman type. Kyungpook Math. J. 54(4), 555-575 (2014)

30. Hilger, S.: Ein maßkettenkalkül mit anwendung auf zentrumsmannigfaltigkeiten. Ph.D. thesis, Universität Würzburg (1988)

31. Hilscher, R.: A time scales version of a Wirtinger-type inequality and applications. J. Comput. Appl. Math. 141(1-2), 219-226 (2002)

32. Karpuz, B., Özkan, U.M.: Some generalizations for Opial's inequality involving several functions and their derivatives of arbitrary order on arbitrary time scales. Math. Inequal. Appl. 14(1), 79-92 (2011)

33. Kaymakçalan, B., Karpuz, B., Öcalan, Ö.: A generalization of Opial's inequality and applications to second-order dynamic equations. Differ. Equ. Dyn. Syst. 18, 11-18 (2010)

34. Li, J.D.: Opial-type integral inequalities involving several higher order derivatives. J. Math. Anal. Appl. 167(1), 98-110 (1992)

35. Li, W.N.: Some delay integral inequalities on time scales. Comput. Math. Appl. 59(6), 1929-1936 (2010)

36. Opial, Z., Sur une inégalité. Ann. Pol. Math. 12(8), 29-32 (1960)

37. Řehák, P.: Hardy inequality on time scales and its application to half-linear dynamic equations. J. Inequal. Appl. 5 $942973(2005)$

38. Řehák, P.: Opial and Lypaunov type inequalities for half-linear dynamic equations. Dyn. Syst. Appl. 13, 445-458 (2014)

39. Saker, S.H., El-Deeb, A.A., Rezk, H.M., Agarwal, R.P.: On Hilbert's inequality on time scales. Appl. Anal. Discrete Math. 11(2), 399-423 (2017)

40. Srivastava, H.M., Tseng, K.L., Tseng, S.J., Lo, J.C.: Some generalizations of Maroni's inequality on time scales. Math Inequal. Appl. 14, 469-480 (2011)

41. Tian, Y., El-Deeb, A.A., Meng, F.: Some nonlinear delay Volterra-Fredholm type dynamic integral inequalities on time scales. Discrete Dyn. Nat. Soc. 2018, Article ID 5841985 (2018)

42. Tseng, S.J., Srivastava, H.M., Tseng, K.L., Lo, J.C.: Some weighted Opial-type inequalities on time scales. Taiwan. J. Math. 14(1), 107-122 (2010)

43. Wong, F.-H., Lian, W.-C., Yu, S.-L., Yeh, C.C.: Some generalizations of Opial's inequalities on time scales. Taiwan. J. Math. 12(2), 463-471 (2008)

44. Yang, G.S.: On a certain result of Z. Opial. Proc. Jpn. Acad. 42, 78-83 (1966) 